\title{
Sex Differences in the Associations of Testosterone and Sex Hormone-Binding Globulin With Metabolic Syndrome in Middle-Aged and Elderly Koreans - The Namwon Study -
}

\author{
Sun-Seog Kweon, MD, PhD; Min-Ho Shin, MD, PhD; Hae-Sung Nam, MD, PhD; \\ Seul-Ki Jeong, MD, PhD; Kyeong-Soo Park, MD, PhD; \\ Jin-Su Choi, MD, PhD; Young-Hoon Lee, MD, PhD
}

\begin{abstract}
Background: We investigated the sex-dependent associations of testosterone and sex hormone-binding globulin (SHBG) levels with metabolic syndrome (MetS).

Methods and Results: We conducted a cross-sectional study of 9,424 community-dwelling adults aged 45-74 years (median age, 63.7 years). MetS was defined according to the updated version of the National Cholesterol Education Program Adult Treatment Panel III criteria. Serum total testosterone (TT) and SHBG levels were determined using a chemiluminescent microparticle immunoassay, and free testosterone (FT) concentrations were calculated. In a multivariate analysis, TT levels were inversely associated with MetS in men (odds ratio [OR] of each standard deviation increase in the logarithmic value, 0.70; 95\% confidence interval $[\mathrm{Cl}], 0.65-0.76$ ), whereas they were positively associated in women $(\mathrm{OR}, 1.17 ; 95 \% \mathrm{Cl}, 1.10-1.24)$. FT levels were positively associated with MetS in women only $(\mathrm{OR}, 1.39 ; 95 \% \mathrm{CI}, 1.30-1.49)$. However, SHBG levels were negatively associated with MetS in both men (OR, 0.57; 95\% Cl, 0.52-0.61) and women (OR, 0.61; 95\% Cl, 0.57-0.66).
\end{abstract}

Conclusions: Our data showed that higher TT levels were associated with a reduced prevalence of MetS in men and an elevated prevalence of MetS in women. Higher SHBG levels were associated with decreased prevalence of MetS in both sexes. These results suggest sex differences in the associations of endogenous testosterone and SHBG with MetS. (Circ J 2013; 77: 734-740)

Key Words: Metabolic syndrome; Sex hormone-binding globulin; Testosterone

$\mathbf{M}$ etabolic syndrome (MetS) is a constellation of metabolic abnormalities that includes abdominal obesity, dyslipidemia, hypertension, and hyperglycemia. MetS is important because of its association with a subsequent high risk of developing diabetes mellitus and cardiovascular disease. ${ }^{1-3}$ Recent epidemiological evidence from cross-sectional studies and meta-analysis indicates that low total testosterone (TT) levels are associated with an increased risk of MetS in men, ${ }^{4-8}$ whereas the opposite is true in women. ${ }^{8-11}$ Sex hormone-binding globulin (SHBG), a major carrier protein of testosterone, is associated with a decreased risk of MetS in both sexes. $4,5,7,8,10-12$ However, epidemiological results on the association between free testosterone (FT) and MetS are inconsistent in men and women., ${ }^{4,9,13}$ Moreover, most of the previous findings have been observed in Caucasians, and only a few community-based studies have reported the association between sex hormones and MetS in Asians. ${ }^{8}$

The aim of this study was to investigate the associations of TT, FT, and SHBG with MetS in community-dwelling middleaged and elderly Korean men and women. We also examined sex differences in the associations of testosterone and SHBG with MetS and its components.

Received May 8, 2012; revised manuscript received September 14, 2012; accepted October 23, 2012; released online November 27, 2012 Time for primary review: 30 days

Department of Preventive Medicine, Chonnam National University Medical School, Gwangju (S.-S.K., M.-H.S., J.-S.C.); Jeonnam Regional Cancer Center, Chonnam National University Hwasun Hospital, Hwasun (S.-S.K.); Department of Preventive Medicine, Chungnam National University College of Medicine, Daejeon (H.-S.N.); Department of Neurology, Chonbuk National University Medical SchoolChonbuk National University Hospital, Jeonju (S.-K.J.); Department of Preventive Medicine, Seonam University College of Medicine, Namwon (K.-S.P.); Department of Preventive Medicine \& Institute of Wonkwang Medical Science, Wonkwang University College of Medicine, Iksan (Y.-H.L.); and Regional Cardiocerebrovascular Center, Wonkwang University Hospital, Iksan (Y.-H.L.), South Korea

Mailing address: Young-Hoon Lee, MD, PhD, Department of Preventive Medicine, Wonkwang University College of Medicine, 344-2 Shinyong-dong, Iksan, Jeollabukdo 570-711, South Korea. E-mail: lyh8275@hanmail.net

ISSN-1346-9843 doi:10.1253/circj.CJ-12-0613

All rights are reserved to the Japanese Circulation Society. For permissions, please e-mail: cj@j-circ.or.jp 


\begin{tabular}{|c|c|c|c|c|c|c|}
\hline & \multicolumn{3}{|c|}{ Men $(n=4,081)$} & \multicolumn{3}{|c|}{ Women $(n=5,343)$} \\
\hline & $\begin{array}{c}\text { MetS (-) } \\
(n=2,461)\end{array}$ & $\begin{array}{c}\text { MetS (+) } \\
(n=1,620)\end{array}$ & $P$ value & $\begin{array}{c}\text { MetS (-) } \\
(n=2,510)\end{array}$ & $\begin{array}{c}\text { MetS }(+) \\
(n=2,833)\end{array}$ & $P$ value \\
\hline Age, years & $62.6 \pm 7.7$ & $61.6 \pm 7.5$ & $<0.001$ & $63.1 \pm 6.7$ & $63.5 \pm 6.3$ & 0.001 \\
\hline $\mathrm{BMI}, \mathrm{kg} / \mathrm{m}^{2}$ & $24.4 \pm 3.1$ & $24.4 \pm 3.1$ & 0.971 & $24.4 \pm 3.1$ & $24.4 \pm 3.1$ & 0.651 \\
\hline $\mathrm{WC}, \mathrm{cm}$ & $82.4 \pm 6.8$ & $89.9 \pm 7.5$ & $<0.001$ & $82.7 \pm 8.4$ & $90.3 \pm 7.8$ & $<0.001$ \\
\hline Systolic BP, mmHg & $122.9 \pm 16.5$ & $132.9 \pm 16.5$ & $<0.001$ & $120.4 \pm 17.6$ & $131.9 \pm 18.8$ & $<0.001$ \\
\hline Diastolic BP, mmHg & $78.9 \pm 9.5$ & $84.3 \pm 9.9$ & $<0.001$ & $77.4 \pm 9.5$ & $82.7 \pm 10.5$ & $<0.001$ \\
\hline $\mathrm{TC}, \mathrm{mg} / \mathrm{dl}$ & $178.9 \pm 33.7$ & $186.3 \pm 38.7$ & $<0.001$ & $192.7 \pm 36.1$ & $199.3 \pm 38.1$ & $<0.001$ \\
\hline $\mathrm{TG}, \mathrm{mg} / \mathrm{dl}$ & $121.2 \pm 74.4$ & $234.3 \pm 154.8$ & $<0.001$ & $112.7 \pm 64.7$ & $196.3 \pm 118.9$ & $<0.001$ \\
\hline $\mathrm{HDL}-\mathrm{C}, \mathrm{mg} / \mathrm{dl}$ & $50.0 \pm 12.1$ & $40.8 \pm 10.0$ & $<0.001$ & $53.1 \pm 11.8$ & $43.2 \pm 9.2$ & $<0.001$ \\
\hline $\mathrm{FBG}, \mathrm{mg} / \mathrm{dl}$ & $102.4 \pm 21.0$ & $117.4 \pm 30.8$ & $<0.001$ & $96.1 \pm 15.5$ & $108.8 \pm 25.4$ & $<0.001$ \\
\hline CRP, mg/dl & $0.06(0.03-0.16)$ & $0.09(0.05-0.19)$ & $<0.001$ & $0.04(0.02-0.10)$ & $0.08(0.04-0.16)$ & $<0.001$ \\
\hline Fasting serum insulin, $\mu \mathrm{U} / \mathrm{ml}$ & $3.2(2.1-4.8)$ & $5.7(4.0-8.0)$ & $<0.001$ & $3.8(2.7-5.4)$ & $6.0(4.2-8.5)$ & $<0.001$ \\
\hline HOMA-IR & $0.81(0.52-1.23)$ & $1.57(1.07-2.35)$ & $<0.001$ & $0.90(0.62-1.29)$ & $1.54(1.07-2.31)$ & $<0.001$ \\
\hline Heart disease & $100(4.1)$ & $91(5.6)$ & 0.021 & $83(3.3)$ & $135(4.8)$ & 0.007 \\
\hline Cerebrovascular disease & $92(3.7)$ & $79(4.9)$ & 0.076 & $58(2.3)$ & $136(4.8)$ & $<0.001$ \\
\hline Antihypertensive medications & $288(11.7)$ & $555(34.3)$ & $<0.001$ & $253(10.1)$ & $1,026(36.2)$ & $<0.001$ \\
\hline Antidiabetic medications & $107(4.3)$ & $253(15.6)$ & $<0.001$ & $52(2.1)$ & $325(11.5)$ & $<0.001$ \\
\hline Education level & & & $<0.001$ & & & 0.018 \\
\hline$\leq 6$ years & $1,579(64.2)$ & $890(55.0)$ & & $2,227(88.7)$ & $2,574(90.9)$ & \\
\hline $7-12$ years & $754(30.6)$ & $621(38.3)$ & & $259(10.3)$ & $244(8.6)$ & \\
\hline$\geq 13$ years & $128(5.2)$ & $109(6.7)$ & & $24(1.0)$ & $15(0.5)$ & \\
\hline Marital status & & & 0.921 & & & 0.951 \\
\hline Married & $2,317(94.1)$ & $1,524(94.1)$ & & $1,827(72.8)$ & $2,060(72.7)$ & \\
\hline Other ${ }^{*}$ & $144(5.9)$ & $96(5.9)$ & & $683(27.2)$ & $773(27.3)$ & \\
\hline Smoking status & & & $<0.001$ & & & 0.001 \\
\hline Never smoker & $495(20.1)$ & $365(22.5)$ & & 2,341 (93.3) & $2,685(94.8)$ & \\
\hline Former smoker & $1,090(44.3)$ & 781 (48.2) & & $43(1.7)$ & $61(2.2)$ & \\
\hline Current smoker & $876(35.6)$ & $474(29.3)$ & & $126(5.0)$ & $87(3.0)$ & \\
\hline Alcohol consumption & & & $<0.001$ & & & $<0.001$ \\
\hline Never drinker & $501(20.4)$ & $227(14.0)$ & & $1,500(59.8)$ & $1,677(59.2)$ & \\
\hline Former drinker & $408(16.6)$ & $265(16.4)$ & & $133(5.3)$ & $240(8.5)$ & \\
\hline Current drinker & $1,552(63.0)$ & $1,128(69.6)$ & & 877 (34.9) & 916 (32.3) & \\
\hline \multicolumn{7}{|l|}{ Sex hormones } \\
\hline $\mathrm{TT}, \mathrm{ng} / \mathrm{ml}$ & $5.85 \pm 2.42$ & $4.98 \pm 1.64$ & $<0.001$ & $0.26 \pm 0.48$ & $0.29 \pm 0.45$ & 0.020 \\
\hline $\mathrm{FT}, \mathrm{ng} / \mathrm{dl}$ & $7.88 \pm 2.82$ & $7.92 \pm 2.10$ & 0.580 & $0.31 \pm 0.54$ & $0.43 \pm 0.71$ & $<0.001$ \\
\hline SHBG, nmol/L & $59.11 \pm 23.03$ & $46.61 \pm 18.20$ & $<0.001$ & $76.87 \pm 29.80$ & $58.81 \pm 25.04$ & $<0.001$ \\
\hline
\end{tabular}

Data are mean \pm standard deviation, medians (interquartile range) or $\mathrm{n}(\%)$.

*Unmarried, divorced, separated, or widowed.

P-values were obtained by Student's t-test or the Mann-Whitney U-test for continuous variables, and the $X^{2}$ test for categorical variables.

BMI, body mass index; BP, blood pressure; CRP, C-reactive protein; FBG, fasting blood glucose; FT, free testosterone; HDL-C, high-density lipoprotein cholesterol; HOMA-IR, homeostatic model assessment-insulin resistance; MetS, metabolic syndrome; SHBG, sex hormone-binding globulin; TC, total cholesterol; TG, triglycerides; TT, total testosterone; WC, waist circumference.

\section{Methods}

\section{Subjects}

The study population consisted of community-dwelling men and women who participated in the baseline survey of the Namwon study, which is an ongoing prospective populationbased cohort study designed to investigate the occurrence and progression of cardiovascular disease, osteoporosis, and dementia in community-dwelling adults aged $45-74$ years. ${ }^{14} \mathrm{~A}$ total of 10,667 subjects (4,201 men and 6,466 women; response rate, $32.3 \%$ ) were enrolled from 2004 to 2007. Of these, 110 subjects were excluded because of missing information about endogenous sex hormones. Moreover, 1,031 premenopausal and perimenopausal women with estradiol $\geq 30 \mathrm{pg} / \mathrm{ml}$ were also excluded to rule out an endogenous estrogenic effect. In total, 9,424 subjects (4,081 men and 5,343 postmenopausal women) were included in the analyses after further exclusion of 102 subjects who lacked information regarding anthropometric or metabolic measures.

This study was conducted in accordance with the Declaration of Helsinki guidelines. All subjects were fully informed of the study content and gave written informed consent for use of their data. The study was approved by the Institutional Review Board of Chonnam National University Hospital.

\section{Testosterone and SHBG}

Serum TT and SHBG levels were determined by a chemiluminescent microparticle immunoassay using the automatic 


\begin{tabular}{|lcrr|}
\hline \multicolumn{2}{l}{ Table 2. Prevalence of MetS and Its Individual Components } & & \\
& Men $(\mathbf{n}=\mathbf{4 , 0 8 1})$ & Women $(\mathbf{n = 5 , 3 4 3 )}$ & P value \\
MetS ( $\geq \mathbf{3}$ components) [metabolic components $\left.{ }^{\star}\right]$ & $1,620(39.7)$ & $2,833(53.0)$ & $<0.001$ \\
High BP & $2,298(56.3)$ & $2,899(54.3)$ & 0.047 \\
Elevated fasting glucose & $2,475(60.6)$ & $2,459(46.0)$ & $<0.001$ \\
Hypertriglyceridemia & $1,729(42.4)$ & $2,182(40.8)$ & 0.136 \\
Low HDL-C & $1,286(31.5)$ & $3,203(59.9)$ & $<0.001$ \\
Abdominal obesity & $1,119(27.4)$ & $3,155(59.0)$ & $<0.001$ \\
No. of metabolic components & & & $<0.001$ \\
0 & $423(10.4)$ & $343(6.4)$ & \\
1 & $905(22.2)$ & $849(15.9)$ & \\
2 & $1,133(27.8)$ & $1,318(24.7)$ & \\
3 & $917(22.5)$ & $1,362(25.5)$ & \\
4 & $530(13.0)$ & $1,028(19.2)$ & \\
5 & $173(4.2)$ & $443(8.3)$ & \\
\hline
\end{tabular}

Data are $n(\%)$.

*High BP (systolic BP $\geq 130 \mathrm{mmHg}$ and/or diastolic BP $\geq 85 \mathrm{mmHg}$ and/or use of medication for hypertension); elevated fasting glucose ( $\geq 100 \mathrm{mg} / \mathrm{dl}$ and/or use of medication for hyperglycemia); hypertriglyceridemia $(\geq 150 \mathrm{mg} / \mathrm{dl})$; low $\mathrm{HDL}-\mathrm{C}(<40 \mathrm{mg} / \mathrm{dl}$ for men and $<50 \mathrm{mg} / \mathrm{dl}$ for women); abdominal obesity (WC $\geq 90 \mathrm{~cm}$ in men and $\geq 85 \mathrm{~cm}$ in women). Abbreviations as in Table 1.

Abbott ARCHITECT System (Abbott Laboratories, Abbott Park, IL, USA). FT concentrations were calculated by the Vermeulen formula..$^{15}$ The detection limits of the assays were $0.08 \mathrm{ng} / \mathrm{ml}$ for TT and $0.1 \mathrm{nmol} / \mathrm{L}$ for SHBG.

\section{MetS Components and Covariates}

Information on cigarette use, alcohol consumption, and medications of each subject was assessed with a standardized questionnaire administered by research staff. All participants underwent a standardized physical examination performed by experienced research staff. Anthropometric measurements, such as height, weight, and waist and hip circumferences, were conducted with the subject standing. Body mass index (BMI) was calculated as weight $(\mathrm{kg})$ divided by height squared $\left(\mathrm{m}^{2}\right)$. Waist circumference (WC) was measured to the nearest $0.1 \mathrm{~cm}$ at the midpoint between the lower border of the rib cage and the upper hip bone during expiration, with the subject wearing no underwear. Blood pressure (BP) was measured with an appropriate-size cuff on the right upper arm using a standard mercury sphygmomanometer (Baumanometer; WA Baum Co Inc, Copiague, NY, USA) after a 5-min rest in the sitting position. Three consecutive systolic and diastolic BP readings, determined to the nearest $2 \mathrm{mmHg}$, were recorded at an interval of $1 \mathrm{~min}$, and the average was used for analysis.

Blood samples were collected from an antecubital vein of each subject in the morning after a 12-h overnight fast. Serum was separated from the samples on-site and stored at $-70^{\circ} \mathrm{C}$ until analysis, all within a 4-week period. Serum total cholesterol, high-density lipoprotein cholesterol (HDL-C), triglycerides, and fasting blood glucose levels were measured using enzymatic techniques. All samples were analyzed using an automatic analyzer (Hitachi-7600 chemical analyzer; Hitachi Ltd, Tokyo, Japan). C-reactive protein (CRP) levels were determined by particle-enhanced immunonephelometry using a BN II nephelometer (Dade Behring, Marburg, Germany). Serum insulin concentrations were measured using a microparticle enzyme immunoassay on the Abbott AXSYM System (Abbott Laboratories). Homeostatic model assessment of insulin resistance (HOMA-IR), a insulin resistance score, was computed with the formula: fasting insulin $(\mu \mathrm{U} / \mathrm{ml}) \times$ fasting glucose (mg/dl) divided by 405 .

\section{MetS Definition}

According to the National Cholesterol Education Program Adult Treatment Panel III (NCEP-ATP III), MetS is identified by the presence of 3 or more of the following 5 conditions: abdominal obesity (WC $\geq 90 \mathrm{~cm}$ for men and $\geq 85 \mathrm{~cm}$ for women), hypertriglyceridemia ( $\geq 150 \mathrm{mg} / \mathrm{dl})$, low HDL-C $(<40 \mathrm{mg} / \mathrm{dl}$ for men and $<50 \mathrm{mg} / \mathrm{dl}$ for women), high BP (systolic $\geq 130 \mathrm{mmHg}$ or diastolic $\geq 85 \mathrm{mmHg}$ or current use of antihypertensive medication), and elevated fasting glucose ( $\geq 100 \mathrm{mg} / \mathrm{dl}$ or current use of hypoglycemic agents). ${ }^{16}$ We defined abdominal obesity using criteria suggested by the Korean Society for the Study of Obesity. ${ }^{17} \mathrm{We}$ applied a new cutoff point for impaired fasting blood glucose as defined by the American Diabetes Association. ${ }^{16}$

\section{Statistical Analysis}

Differences in continuous variables and in the proportion of categorical variables for subjects with and without MetS were compared using Student's t-test and the $\chi^{2}$ test, respectively. The $\chi^{2}$ test was used to assess sex differences in the prevalence of MetS and its components. The relationship between MetS and sex hormones was analyzed using multiple logistic regression. TT, FT, and SHBG were categorized into quartiles. Odds ratios (OR) are presented with $95 \%$ confidence intervals (CI). Additionally, because of skewed sex hormone distributions, the associations of continuous logarithmic values of TT, FT, and SHBG with MetS and its components were analyzed using multiple logistic regression. In the multiple logistic regression analysis, we sequentially adjusted for several potential confounding factors including age, education level, marital status, smoking status, alcohol consumption, heart disease, cerebrovascular disease, and HOMA-IR. Because emerging laboratory and clinical results have shown a strong relationship between CRP, a chronic low-grade inflammation biomarker, and MetS and its components, ${ }^{18}$ we further adjusted the analysis for CRP. The estimates for the associations of testosterone and SHBG with MetS were adjusted in 3 models: model 1 was adjusted for age; model 2 was further adjusted for education level, marital status, smoking status, alcohol consumption, heart disease, cerebrovascular disease, and CRP; model 3 was additionally adjusted for HOMA-IR. All statistical analyses were 


\begin{tabular}{|c|c|c|c|}
\hline & $\begin{array}{c}\text { Model } 1 \\
\text { OR }(95 \% \mathrm{Cl})\end{array}$ & $\begin{array}{c}\text { Model } 2 \\
\text { OR (95\% Cl) }\end{array}$ & $\begin{array}{c}\text { Model } 3 \\
\text { OR }(95 \% \mathrm{Cl})\end{array}$ \\
\hline \multicolumn{4}{|l|}{ Men $(n=4,081)$} \\
\hline \multicolumn{4}{|l|}{$\mathrm{TT}, \mathrm{ng} / \mathrm{ml}$} \\
\hline $\log [T T],+1 S D$ & $0.62(0.57-0.67)$ & $0.62(0.58-0.68)$ & $0.71(0.65-0.77)$ \\
\hline $1 Q(\leq 4.25)$ & 1.00 & 1.00 & 1.00 \\
\hline $2 Q(4.26-5.30)$ & $0.61(0.51-0.73)$ & $0.61(0.51-0.72)$ & $0.71(0.59-0.85)$ \\
\hline $3 Q(5.31-6.48)$ & $0.41(0.35-0.49)$ & $0.41(0.34-0.49)$ & $0.50(0.41-0.61)$ \\
\hline $4 Q(\geq 6.49)$ & $0.29(0.24-0.36)$ & $0.30(0.25-0.36)$ & $0.39(0.31-0.47)$ \\
\hline \multicolumn{4}{|l|}{ FT, ng/dl } \\
\hline $\log [\mathrm{FT}],+1 \mathrm{SD}$ & $0.97(0.91-1.04)$ & $0.97(0.91-1.04)$ & $1.00(0.93-1.07)$ \\
\hline $1 \mathrm{Q}(\leq 6.63)$ & 1.00 & 1.00 & 1.00 \\
\hline 2Q (6.64-7.81) & $1.04(0.88-1.25)$ & $1.06(0.89-1.27)$ & $1.12(0.92-1.37)$ \\
\hline $3 Q(7.82-9.06)$ & $0.98(0.81-1.17)$ & $0.98(0.81-1.18)$ & $1.04(0.86-1.27)$ \\
\hline $4 Q(\geq 9.07)$ & $1.03(0.86-1.24)$ & $1.04(0.86-1.26)$ & $1.07(0.87-1.31)$ \\
\hline \multicolumn{4}{|l|}{ SHBG, nmol/L } \\
\hline Log[SHBG], +1SD & $0.50(0.46-0.54)$ & $0.50(0.47-0.55)$ & $0.57(0.52-0.62)$ \\
\hline $1 \mathrm{Q}(\leq 38.5)$ & 1.00 & 1.00 & 1.00 \\
\hline 2Q (38.6-50.7) & $0.52(0.44-0.63)$ & $0.52(0.44-0.63)$ & $0.60(0.50-0.73)$ \\
\hline $3 Q(50.8-65.8)$ & $0.35(0.29-0.43)$ & $0.35(0.29-0.42)$ & $0.41(0.34-0.50)$ \\
\hline $4 Q(\geq 65.9)$ & $0.19(0.15-0.23)$ & $0.20(0.16-0.24)$ & $0.26(0.21-0.32)$ \\
\hline \multicolumn{4}{|l|}{ Women $(n=5,343)$} \\
\hline \multicolumn{4}{|l|}{$\mathrm{TT}, \mathrm{ng} / \mathrm{ml}$} \\
\hline $\log [T T],+1 S D$ & $1.22(1.16-1.29)$ & $1.23(1.16-1.30)$ & $1.16(1.09-1.24)$ \\
\hline $1 Q(\leq 0.15)$ & 1.00 & 1.00 & 1.00 \\
\hline $2 Q(0.16-0.22)$ & $1.13(0.97-1.31)$ & $1.13(0.97-1.32)$ & $1.09(0.92-1.29)$ \\
\hline $3 Q(0.23-0.31)$ & $1.41(1.21-1.63)$ & $1.41(1.21-1.64)$ & $1.37(1.16-1.62)$ \\
\hline $4 Q(\geq 0.32)$ & $1.76(1.51-2.04)$ & $1.77(1.51-2.06)$ & $1.36(1.15-1.62)$ \\
\hline \multicolumn{4}{|l|}{ FT, ng/dl } \\
\hline $\log [\mathrm{FT}],+1 \mathrm{SD}$ & $1.61(1.52-1.71)$ & $1.62(1.52-1.72)$ & $1.39(1.30-1.48)$ \\
\hline $1 \mathrm{Q}(\leq 0.17)$ & 1.00 & 1.00 & 1.00 \\
\hline $2 Q(0.18-0.28)$ & $1.52(1.30-1.77)$ & $1.54(1.32-1.80)$ & $1.41(1.19-1.67)$ \\
\hline $3 Q(0.29-0.44)$ & $2.25(1.93-2.63)$ & 2.27 (1.94-2.66) & $1.88(1.58-2.23)$ \\
\hline $4 Q(\geq 0.45)$ & $3.82(3.24-4.50)$ & $3.84(3.25-4.53)$ & $2.36(1.97-2.83)$ \\
\hline \multicolumn{4}{|l|}{ SHBG, nmol/L } \\
\hline Log[SHBG], +1SD & $0.48(0.45-0.51)$ & $0.48(0.45-0.51)$ & $0.61(0.57-0.66)$ \\
\hline $1 \mathrm{Q}(\leq 46.2)$ & 1.00 & 1.00 & 1.00 \\
\hline $2 Q(46.3-62.7)$ & $0.49(0.41-0.58)$ & $0.49(0.42-0.58)$ & $0.70(0.58-0.83)$ \\
\hline $3 Q(62.8-84.1)$ & $0.32(0.27-0.38)$ & $0.32(0.27-0.38)$ & $0.53(0.44-0.63)$ \\
\hline $4 \mathrm{Q}(\geq 84.2)$ & $0.15(0.12-0.17)$ & $0.15(0.12-0.18)$ & $0.28(0.23-0.34)$ \\
\hline
\end{tabular}

Model 1 was adjusted for age.

Model 2 was adjusted for age, education level, marital status, smoking status, alcohol consumption, heart disease, cerebrovascular disease, and CRP.

Model 3 was adjusted for age, education level, marital status, smoking status, alcohol consumption, heart disease, cerebrovascular disease, CRP, and HOMA-IR.

$\mathrm{Cl}$, confidence interval; OR, odds ratio. Other abbreviations as in Table 1.

performed using SPSS version 18.0 (SPSS, Chicago, IL, USA).

\section{Results}

\section{Baseline Characteristics and MetS Prevalence}

Table 1 shows the general characteristics of the study population according to the presence or absence of MetS. Significant differences were observed between subjects with MetS and those without MetS for all characteristics in both men and women, with the exception of marital status and BMI. Men with MetS had significantly lower TT levels than those with- out MetS ( $\mathrm{P}<0.001$ for each), whereas women with MetS had significantly higher TT and FT levels than those without MetS. Both men and women with MetS had significantly lower SHBG levels than those without MetS (all $\mathrm{P}<0.001$ ).

MetS was present in $39.7 \%$ of men and $53.0 \%$ of women $(\mathrm{P}<0.001$ each). Among the men, elevated fasting glucose was the most common MetS component (60.6\%), followed by high $\mathrm{BP}$, hypertriglyceridemia, low HDL-C, and abdominal obesity. Low HDL-C was the most common MetS component $(59.9 \%)$ in women, followed by abdominal obesity, high BP, elevated fasting glucose, and hypertriglyceridemia (Table 2). 
Table 4. Adjusted OR (95\% Cl) for the Risk of Metabolic Components to a 1 SD Increase in Log[TT], Log[FT], and Log[SHBG]

\begin{tabular}{lccccc} 
& High BP & $\begin{array}{c}\text { Elevated fasting } \\
\text { glucose }\end{array}$ & Hypertriglyceridemia & Low HDL-C & $\begin{array}{c}\text { Abdominal } \\
\text { obesity }\end{array}$ \\
$\begin{array}{l}\text { Men }(\mathbf{n}=4, \mathbf{0 8 1}) \\
\text { Log[TT], ng/ml }\end{array}$ & $0.93(0.86-0.99)$ & $0.91(0.85-0.98)$ & $0.80(0.75-0.86)$ & $0.84(0.79-0.91)$ & $0.80(0.74-0.86)$ \\
Log[FT], ng/dl & $1.04(0.97-1.11)$ & $0.97(0.90-1.04)$ & $1.15(1.07-1.23)$ & $0.85(0.79-0.91)$ & $1.01(0.94-1.09)$ \\
$\quad$ Log[SHBG], nmol/L & $0.83(0.77-0.89)$ & $0.90(0.83-0.98)$ & $0.55(0.51-0.60)$ & $0.96(0.89-1.04)$ & $0.64(0.58-0.69)$ \\
Women $(\mathbf{n}=5,343)$ & & & & & \\
$\quad$ Log[TT], ng/ml & $1.09(1.03-1.16)$ & $1.16(1.09-1.24)$ & $0.99(0.93-1.05)$ & $0.98(0.92-1.03)$ & $1.21(1.13-1.28)$ \\
Log[FT], ng/dl & $1.14(1.07-1.21)$ & $1.21(1.14-1.30)$ & $1.16(1.09-1.23)$ & $1.02(0.96-1.08)$ & $1.45(1.36-1.55)$ \\
Log[SHBG], nmol/L & $0.88(0.83-0.94)$ & $0.87(0.81-0.93)$ & $0.66(0.62-0.71)$ & $0.88(0.83-0.94)$ & $0.59(0.55-0.63)$ \\
\hline
\end{tabular}

Adjusted for age, education level, marital status, smoking status, alcohol consumption, heart disease, cerebrovascular disease, CRP, HOMA$\mathrm{IR}$, and other MetS components.

SD, standard deviation. Other abbreviations as in Tables 1,4.

\section{MetS According to TT, FT, and SHBG}

The association of TT, FT, and SHBG with MetS as determined by multiple logistic regression analysis is shown in Table 3. In men, the ORs of MetS for the second, third, and fourth quartiles of TT $(0.61$ [95\% CI: $0.51-0.73], 0.41$ [95\% CI: $0.35-0.49$ ], and 0.29 [95\% CI: 0.24-0.36], respectively) decreased significantly when compared with the lowest TT quartile, after adjustment for age. After additional adjustment for smoking, alcohol consumption, and CRP (model 2), the inverse associations remained significant. After further adjustment for HOMA-IR (model 3), the inverse associations were attenuated slightly but remained significant. In contrast, the ORs of MetS for the third and fourth quartiles of TT in women increased significantly compared with the lowest quartile of TT in all models. No significant association was found between FT and MetS in men. However, significant positive associations were observed between FT and the MetS in women in models 1, 2, and 3. Compared with the lowest SHBG quartile, in the multivariate analysis, the ORs of MetS for the second, third, and fourth quartiles of TT decreased significantly in both men (0.60 [95\% CI: 0.50-0.73], 0.41 [95\% CI: 0.34-0.50], and 0.26 [95\% CI: $0.21-0.32$ ], respectively) and women $(0.70$ [95\% CI: 0.58-0.83], 0.53 [95\% CI: 0.44-0.63], and 0.28 [95\% CI: 0.23-0.34], respectively).

The associations of continuous TT, FT, and SHBG with MetS are also shown in Table 3. In men, each standard deviation (SD) increase in the logarithmic value of TT was significantly associated with a decreased OR of MetS (0.62 [95\% CI: 0.57-0.67]), after adjustment for age. After further adjustment for several risk factors (model 3), this association was attenuated slightly but remained significant, with a decreased OR (0.71 [95\% CI: 0.65-0.77]). In women, each SD increase in the logarithmic values of TT and FT was positively associated with an increased OR (1.22 [95\% CI: 1.16-1.29] and 1.61 [95\% CI: $1.52-1.71]$, respectively), in the age-adjusted analysis, and the associations were attenuated but remained significant, with an increased OR (1.16 [95\% CI: 1.09-1.24] and 1.39 [95\% CI: 1.30-1.48], respectively), after further adjustment for several risk factors (model 3). SHBG was significantly associated with MetS in both sexes in all models. In model 3, each SD increase in the logarithmic value of SHBG was significantly associated with a decreased OR (0.57 [95\% CI: 0.52-0.62] and 0.61 [95\% CI: 0.57-0.66], respectively) of MetS in both men and women. When the premenopausal and perimenopausal women with estradiol levels $\geq 30 \mathrm{pg} / \mathrm{ml}$ (1,031 women) were included in the analysis, the strength of the associations of testosterone and SHBG with MetS were only slightly changed (Table S1).

\section{MetS Components According to TT, FT, and SHBG}

The associations between the continuous logarithmic values of TT, FT, and SHBG and the MetS components after adjusting for covariates are shown in Table 4. In men, TT levels were negatively associated with all MetS components. FT levels were negatively associated with only low HDL-C and positively associated with only hypertriglyceridemia in men. In women, TT levels were positively associated with high BP, elevated fasting glucose, and abdominal obesity. FT levels were positively associated with all MetS components, except low HDL-C, in women. SHBG levels were negatively associated with all MetS components, except low HDL-C, in men and all MetS components in women.

\section{Discussion}

Our population-based cross-sectional study of communitydwelling middle-aged and elderly Koreans examined sexdependent associations between endogenous testosterone levels and MetS. TT levels were negatively associated with MetS in men, but positively associated with MetS in women. FT levels were positively associated with MetS in women only. SHBG was inversely associated with MetS in both sexes and no sex-specific association was observed.

Previous epidemiological studies have shown that higher TT levels are associated with a reduced MetS OR in men, ${ }^{4-7}$ but with an increased OR in women. ${ }^{9-11}$ In a recent meta-analysis, ${ }^{8}$ men in the highest TT tertile had a decreased OR of MetS compared with those in the lowest tertile, whereas women in the highest TT tertile had an increased OR of MetS compared with those in the lowest tertile. Our results confirm a sex difference in the association between TT and MetS, which is consistent with the findings of previous studies. ${ }^{4-11}$ However, recent longitudinal studies have shown a sex difference in the association between TT and incident MetS; no association in men, ${ }^{12}$ but a positive association in women. ${ }^{19}$

Additionally, studies have shown that men with lower TT levels have an increased OR of having MetS components than those with higher TT levels, independent of several cardiovascular risk factors. ${ }^{4,6,7,13}$ Low levels of TT have been associated with individual components of MetS, such as abdominal obesity, ${ }^{4,6,20}$ dyslipidemia, ${ }^{4,5,20,21}$ hypertension, ,,22 and high glucose/ insulin resistance. ${ }^{6,20,23,24}$ Our results suggest that low TT levels are associated with all MetS components in men. However, low TT levels were strongly associated with abdominal obesity and dyslipidemia, but were only weakly associated with high BP and elevated fasting glucose levels. Previous reports 
have shown similar patterns of association between TT and MetS components. ${ }^{5,20}$ In women, relatively few studies have reported independent associations among TT levels, MetS, ${ }^{9-11}$ and MetS components. ${ }^{9}$

The complex biological mechanisms underlying the sexspecific relationship between TT and MetS are not fully understood. Evidence from animal studies has shown sex-specific effects of TT on MetS components. Low TT levels were associated with dyslipidemia and insulin resistance in castrated male rats, ${ }^{25,26}$ whereas exposure to exogenous TT resulted in insulin resistance and other MetS components in female rats. ${ }^{27,28}$ Therefore, we adjusted for HOMA in model 3 to examine the extent to which the relationship between TT and MetS could be accounted for by insulin resistance. The strength of the association was slightly attenuated, but remained statistically significant after adjusting for HOMA, suggesting that the effects were partially mediated by insulin resistance. A previous study found that the association between testosterone and MetS is bidirectional and suggested that MetS is not only the consequence of low testosterone levels, but also a cause of low TT and FT levels in middle-aged men. ${ }^{29}$ Moreover, the Framingham study recently showed that although cross-sectional associations suggest that testosterone and SHBG are related to MetS, only SHBG remains associated with MetS in longitudinal analyses. ${ }^{12}$ Thus, changes in TT, FT, and SHBG may be a consequence of the metabolic disorders that characterize MetS, instead of determinants of these changes. Further prospective epidemiological studies are needed to determine the temporality of this association.

Unlike TT, FT was not significantly associated with MetS in men. The literature on the association between FT and MetS in men is inconsistent. Some epidemiological studies have found a significant inverse association between FT and MetS in men, ${ }^{7}$ whereas other studies have shown that FT is not associated with MetS in men., ${ }^{43}$ The Framingham Heart Study showed that the inverse relationship between FT and MetS in men was no longer significant after adjusting for age, BMI, and insulin sensitivity. ${ }^{12}$ In women, significant dose-response associations between FT and MetS and all its components, except low HDL-C, were observed in our study. However, a recent study found no significant association between FT and MetS in women. ${ }^{9}$ Some studies have found a significant relationship between MetS and the free androgen index, which is the ratio of TT/SHBG $\times 100$, and is highly correlated with FT. ${ }^{10,11}$

Epidemiological studies have established that lower SHBG levels are independently associated with an increased OR of MetS in both men ${ }^{4,5,7}$ and women. ${ }^{10,11,30}$ Most studies found inverse dose-response relationships between SHBG and MetS, without a sex-specific association. The results of our study were consistent with previous findings showing a negative, dose-response association between SHBG and MetS in both sexes. Moreover, recent studies found that low SHBG levels were positively associated with incident MetS in both sexes. ${ }^{12,19}$ The associations between SHBG levels and MetS as well as TT were independently significant. Previous epidemiological studies have reported that the association between FT and MetS is less consistent than that for TT in men.,8 These findings suggest that the association between SHBG and MetS is more etiologically important than the relationship between TT and MetS. However, the biological mechanism underlying the association between SHBG and MetS is not completely understood. It is unclear whether SHBG is an early predictor of MetS or an additional component of MetS. ${ }^{28}$ When we further adjusted for HOMA-IR, the inverse associations were attenuated slightly but remained significant in both men and women. Because portal insulin concentrations are a major negative regulator of SHBG production in the liver, insulin resistance could account for the strong and consistent inverse relationship between SHBG levels and MetS. ${ }^{31}$

\section{Study Limitations}

First, our cross-sectional design limited our ability to determine temporal associations of TT, FT, and SHBG with MetS. Second, it is possible that our low response rate $(32.3 \%)$ does not reflect the current status of the entire population. Although this might result in selection bias and limit generalization of the present findings, the age and sex distributions in the present study subjects were comparable with those in the general population (Table S2). Third, misclassification error because of a single anthropometric measurement and a single blood sample might have attenuated the associations of testosterone and SHBG levels with MetS. However, single-point plasma TT levels fairly and reliably reflect typical TT levels. ${ }^{32}$ Fourth, potential confounders such as physical activity, occupation and income were not adjusted for because these variables were not evaluated in the present study.

Nevertheless, our study had some strengths. First, it included a large community-based population of both older men and women. Second, we clearly followed the guidelines for the definition of MetS by the NCEP-ATP III, and all MetS components were examined by direct measurement (BP and WC) and blood sampling after a 12-h overnight fast (glucose, triglycerides, and HDL-C). Third, fasting blood samples were collected only in the morning to minimize any potential effects of circadian variation on TT and SHBG concentrations.

In summary, we found sex-specific associations between sex hormones and MetS as well as its individual components. Lower TT levels were associated with MetS in men, but higher TT and FT levels were associated with MetS in women. Moreover, SHBG levels were negatively associated with MetS in both sexes. Longitudinal studies are needed to clarify the temporality of these associations and their underlying mechanisms.

\section{Acknowledgments}

This work was supported by a National Research Foundation of Korea Grant funded by the Korean Government (KRF-2008-331-E00073). This work was also partially supported by the Korean Centers for Disease Control and Prevention (2004-347-6111-213, 2005-347-2400-2440-215, 2006-347-2400-2440-215, 20070308455-00).

\section{References}

1. Galassi A, Reynolds K, He J. Metabolic syndrome and risk of cardiovascular disease: A meta-analysis. Am J Med 2006; 119: 812-819.

2. Ford ES. Risks for all-cause mortality, cardiovascular disease, and diabetes associated with the metabolic syndrome: A summary of the evidence. Diabetes Care 2005; 28: 1769-1778.

3. Saito I. Epidemiological evidence of type 2 diabetes mellitus, metabolic syndrome, and cardiovascular disease in Japan. Circ J 2012; 76: $1066-1073$.

4. Li C, Ford ES, Li B, Giles WH, Liu S. Association of testosterone and sex hormone-binding globulin with metabolic syndrome and insulin resistance in men. Diabetes Care 2010; 33: 1618-1624.

5. Kupelian V, Hayes FJ, Link CL, Rosen R, McKinlay JB. Inverse association of testosterone and the metabolic syndrome in men is consistent across race and ethnic groups. J Clin Endocrinol Metab 2008; 93: 3403-3410.

6. Muller M, Grobbee DE, den Tonkelaar I, Lamberts SW, van der Schouw YT. Endogenous sex hormones and metabolic syndrome in aging men. J Clin Endocrinol Metab 2005; 90: 2618-2623.

7. Laaksonen DE, Niskanen L, Punnonen K, Nyyssönen K, Tuomainen TP, Valkonen VP, et al. Testosterone and sex hormone-binding globulin predict the metabolic syndrome and diabetes in middle-aged men. Diabetes Care 2004; 27: 1036-1041.

8. Brand JS, van der Tweel I, Grobbee DE, Emmelot-Vonk MH, van der Schouw YT. Testosterone, sex hormone-binding globulin and the 
metabolic syndrome: A systematic review and meta-analysis of observational studies. Int J Epidemiol 2011; 40: 189-207.

9. Patel SM, Ratcliffe SJ, Reilly MP, Weinstein R, Bhasin S, Blackman $\mathrm{MR}$, et al. Higher serum testosterone concentration in older women is associated with insulin resistance, metabolic syndrome, and cardiovascular disease. J Clin Endocrinol Metab 2009; 94: 4776-4784.

10. Weinberg ME, Manson JE, Buring JE, Cook NR, Seely EW, Ridker $\mathrm{PM}$, et al. Low sex hormone-binding globulin is associated with the metabolic syndrome in postmenopausal women. Metabolism 2006; 55: $1473-1480$.

11. Santoro N, Torrens J, Crawford S, Allsworth JE, Finkelstein JS, Gold $\mathrm{EB}$, et al. Correlates of circulating androgens in mid-life women: The study of women's health across the nation. J Clin Endocrinol Metab 2005; 90: 4836-4845.

12. Bhasin S, Jasjua GK, Pencina M, D'Agostino R Sr, Coviello AD, Vasan RS, et al. Sex hormone-binding globulin, but not testosterone, is associated prospectively and independently with incident metabolic syndrome in men: The Framingham heart study. Diabetes Care 2011; 34: 2464-2470.

13. Kupelian V, Page ST, Araujo AB, Travison TG, Bremner WJ, McKinlay JB. Low sex hormone-binding globulin, total testosterone, and symptomatic androgen deficiency are associated with development of the metabolic syndrome in non-obese men. J Clin Endocrinol Metab 2006; 91: 843-850.

14. Nam HS, Shin MH, Zmuda JM, Leung PC, Barrett-Connor E, Orwoll ES, et al. Race/ethnic differences in bone mineral densities in older men. Osteoporos Int 2010; 21: 2115-2123.

15. Vermeulen A, Verdonck L, Kaufman JM. A critical evaluation of simple methods for the estimation of free testosterone in serum. $J$ Clin Endocrinol Metab 1999; 84: 3666-3672.

16. Grundy SM, Cleeman JI, Daniels SR, Donato KA, Eckel RH, Franklin BA, et al; American Heart Association; National Heart, Lung, and Blood Institute. Diagnosis and management of the metabolic syndrome: An American Heart Association/National Heart, Lung, and Blood Institute Scientific Statement. Circulation 2005; 112: $2735-$ 2752.

17. Lee SY, Park HS, Kim DJ, Han JH, Kim SM, Cho GJ, et al. Appropriate waist circumference cutoff points for central obesity in Korean adults. Diabetes Res Clin Pract 2007; 75: 72-80.

18. Devaraj S, Valleggi S, Siegel D, Jialal I. Role of C-reactive protein in contributing to increased cardiovascular risk in metabolic syndrome. Curr Atheroscler Rep 2010; 12: 110-118.

19. Torréns JI, Sutton-Tyrrell K, Zhao X, Matthews K, Brockwell S, Sowers $\mathrm{M}$, et al. Relative androgen excess during the menopausal transition predicts incident metabolic syndrome in midlife women: Study of Women's Health Across the Nation. Menopause 2009; 16: 257-264.

20. Chubb SA, Hyde Z, Almeida OP, Flicker L, Norman PE, Jamrozik K, et al. Lower sex hormone-binding globulin is more strongly associated with metabolic syndrome than lower total testosterone in older men: The Health in Men Study. Eur J Endocrinol 2008; 158: $785-$ 792.

21. Atlantis E, Martin SA, Haren MT, O'Loughlin PD, Taylor AW, Anand-Ivell R, et al. Demographic, physical and lifestyle factors associated with androgen status: The Florey Adelaide Male Ageing Study
(FAMAS). Clin Endocrinol (Oxf) 2009; 71: 261-272.

22. Torkler S, Wallaschofski H, Baumeister SE, Völzke H, Dörr M, Felix $\mathrm{S}$, et al. Inverse association between total testosterone concentrations, incident hypertension and blood pressure. Aging Male 2011; 14: 176182.

23. Ding EL, Song Y, Malik VS, Liu S. Sex differences of endogenous sex hormones and risk of type 2 diabetes: A systematic review and meta-analysis. JAMA 2006; 295: 1288-1299.

24. Atlantis E, Lange K, Martin S, Haren MT, Taylor A, O'Loughlin PD, et al. Testosterone and modifiable risk factors associated with diabetes in men. Maturitas 2011; 68: 279-285.

25. Holmang A, Bjorntorp P. The effects of testosterone on insulin sensitivity in male rats. Acta Physiol Scand 1992; 146: 505-510.

26. Xu XF, De Pergola G, Björntorp P. Testosterone increases lipolysis and the number of beta-adrenoceptors in male rat adipocytes. Endocrinology 1991; 128: 379-382.

27. Nilsson C, Niklasson M, Eriksson E, Björntorp P, Holmäng A. Imprinting of female offspring with testosterone results in insulin resistance and changes in body fat distribution at adult age in rats. $J$ Clin Invest 1998; 101: 74-78.

28. Alexanderson C, Eriksson E, Stener-Victorin E, Lystig T, Gabrielsson B, Lönn M, et al. Postnatal testosterone exposure results in insulin resistance, enlarged mesenteric adipocytes, and an atherogenic lipid profile in adult female rats: Comparisons with estradiol and dihydrotestosterone. Endocrinology 2007; 148: 5369-5376.

29. Laaksonen DE, Niskanen L, Punnonen K, Nyyssönen K, Tuomainen TP, Valkonen VP, et al. The metabolic syndrome and smoking in relation to hypogonadism in middle-aged men: A prospective cohort study. J Clin Endocrinol Metab 2005; 90: 712-719.

30. Maggio M, Lauretani F, Ceda GP, Bandinelli S, Basaria S, Paolisso G, et al. Association of hormonal dysregulation with metabolic syndrome in older women: Data from the InCHIANTI study. Am $J$ Physiol Endocrinol Metab 2007; 292: E353-E358.

31. Yki-Järvinen H, Mäkimattila S, Utriainen T, Rutanen EM. Portal insulin concentrations rather than insulin sensitivity regulate serum sex hormone-binding globulin and insulin-like growth factor binding protein 1 in vivo. J Clin Endocrinol Metab 1995; 80: 3227-3232.

32. Vermeulen A, Verdonck G. Representativeness of a single point plasma testosterone level for the long term hormonal milieu in men. J Clin Endocrinol Metab 1992; 74: 939-942.

\section{Supplementary Files}

Supplementary File 1

Table S1. Relationship Between MetS and Sex Hormones by Multiple Logistic Regression Analyses in Women $(n=6,374)$, Including Premenopausal and Perimenopausal Women With Estradiol $\geq 30 \mathrm{pg} / \mathrm{ml}$

Table S2. Comparison of Sex and Age Distributions Between General Population and Study Participants

Please find supplementary file(s);

http://dx.doi.org/10.1253/circj.CJ-12-0613 